\title{
CORRECTION
}

\section{Correction to: HBV molecular epidemiology and clinical condition of immigrants living in Italy}

\author{
Caterina Sagnelli ${ }^{1,2,3,4} \cdot$ Massimo Ciccozzi $^{5} \cdot$ Loredana Alessio $^{2,3} \cdot$ Eleonora Cella $^{5} \cdot$ Luciano Gualdieri $^{6}$. \\ Mariantonietta Pisaturo ${ }^{2,3,7}$. Carmine Minichini ${ }^{1}$. Giovanni Di Caprio ${ }^{2,3} \cdot$ Mario Starace $^{1} \cdot$ Lorenzo Onorato $^{2,3}$. \\ Mara Caroprese $^{2,3} \cdot$ Laura Occhiello $^{2,3} \cdot$ Silvia Angeletti $^{5} \cdot$ Gaetano Scotto $^{8} \cdot$ Margherita Macera $^{1,2,3}$. \\ Evangelista Sagnelli ${ }^{1}$ (1) Nicola Coppola ${ }^{1}$
}

Published online: 22 October 2019

(c) Springer-Verlag GmbH Germany, part of Springer Nature 2019

\section{Correction to: Infection (2018) 46:523-531 https://doi.org/10.1007/s15010-018-1153-1}

The original version of this article unfortunately contained a mistake. The name of the author Mara Caroprese was rendered wrongly. The correct name is shown above.

The original article can be found online at https://doi.org/10.1007/ s15010-018-1153-1.

\section{Evangelista Sagnelli}

evangelista.sagnelli@unicampania.it;

evangelistasagnelli@libero.it

1 Section of Infectious Diseases, Department of Mental Health and Public Medicine, University of Campania Luigi Vanvitelli, Via L. Armanni 5, 80131 Naples, Italy

2 Medical Center, Centro Sociale ex Canapificio, Caserta, Italy

3 Medical Center, Centro di Accoglienza "La tenda di Abramo", Caserta, Italy

4 Medical Center, Centro Suore Missionarie della Carità, Naples, Italy

5 Unit of Clinical Laboratory Science, University Campus Bio-Medico of Rome, Rome, Italy

6 Medical Center, Centro per la Tutela della Salute degli Immigrati, Naples, Italy

7 Infectious Diseases Unit, AORN Sant'Anna e San Sebastiano, Caserta, Italy

8 Infectious Diseases Unit, Foggia, Italy 